\title{
TESTING THE VECTOR CONDENSATE MODEL OF ELECTROWEAK INTERACTIONS AT HIGH ENERGY HADRON COLLIDERS
}

\author{
G. Cynolter, E. Lendvai and G. Pócsik \\ Institute for Theoretical Physics, \\ Eötvös Lorand University, Budapest
}

\begin{abstract}
In the vector condensate model a doublet of vector fields plays the role of the Higgs doublet of standard model and the gauge symmetry is broken dynamically. This results in a theory surviving the test of radiative corrections provided the new charged and neutral vector particles B have masses of at least several hundred GeV's. In this note we show that while at the Tevatron the heavy B-particle production is too low, at LHC the yield is large and, for instance, the inclusive cross section of $B^{+} B^{-}$pairs is $51.5(15.3) \mathrm{fb}$ at $\sqrt{s}=14 \mathrm{TeV}, m_{B}=$ 400(500) GeV.
\end{abstract}

The vector condensate model employs the Lagrangian of the standard model of electroweak interactions but the usual scalar doublet is replaced by a doublet of vector fields $B_{\mu}[1]$ whose neutral component forms a condensate breaking the gauge symmetry dynamically and providing nonvanishing $W, Z$ and vanishing photon masses. A quartic self-coupling of $B_{\mu}$ gives masses to the B-particles, as well as the interaction of B-pairs with fermions gives rise to fermion masses and embeds the Kobayashi-Maskawa mechanism [1].

Looking at the $\mathrm{S}$ parameter it follows that the B-particles are heavy, at $\Lambda=1 \mathrm{TeV}$ the threshold is about $m_{0} \simeq 400-500 \mathrm{GeV}$ for $B^{0}$ and $m_{+} \simeq 200-350 \mathrm{GeV}$ for $B^{+}[2]$. Higher $\Lambda$ attracts higher minimum masses. 
The allowed regions by $\mathrm{S}$ are tightened by the $\mathrm{T}$ parameter [3]. For example at $\Lambda=1 \mathrm{TeV}, m_{0}=400 \mathrm{GeV}$ the threshold for $m_{+}$is increased to $630 \mathrm{GeV}$. In general, to each momentum scale there exists a range of $\mathrm{B}$ masses where the radiative corrections are suitably small by partial cancellations. This is why $\Lambda$ cannot be too large as compared to B masses.

Recently it has been shown that pairs of heavy B-particles are copiously produced at high energy linear $e^{+} e^{-}$colliders [4]. In the present note we study the production of B-particles at the Tevatron and LHC. We show that producing heavy B-particles at LHC is very favourable having a large cross section while at Tevatron energy the production cross section cannot exceed (0.01-0.02) fb which is far below the discovery limit.

Since fermions are coupled very weakly to B-pairs [1] in the vector condensate model, producing B-pairs is expected to be more considerable from virtual $\gamma$ and $\mathrm{Z}$ exchanges, that is we consider the Drell-Yan mechanism [5], $p(\bar{p}) \rightarrow B \bar{B}+X$ via quark-antiquark annihilation.

The Drell-Yan cross section for the above hadronic collisions can be written as $[5,6]$

$$
\begin{array}{r}
\sigma(p(\bar{p}) \rightarrow B \bar{B}+X)=\int_{\tau_{0}}^{1} d \tau \int_{\tau}^{1} \frac{d x}{2 x} \sum_{i} \sigma\left(q_{i} \bar{q}_{i} \rightarrow B \bar{B}\right) \\
\left(f_{i}^{1}(x, \hat{s}) f_{\bar{i}}^{2}(\tau / x, \hat{s})+f_{\bar{i}}^{1}(x, \hat{s}) f_{i}^{2}(\tau / x, \hat{s})\right)
\end{array}
$$

where $x$ and $\tau / x$ are the parton momentum fractions, $\hat{s}=\tau s$ is the square of the centre of mass energy of $q_{i} \bar{q}_{i}, \mathrm{~s}$ is the same for the hadronic initial state, $f_{i}^{1}(x, \hat{s})$ means the number distribution of $i$ quarks in hadron 1 at the scale $\hat{s}$ and the sum runs over the quark flavours u,d,s,c. In the computation the MRS (G) fit program [7] was used for the parton distributions.

The angle integrated, colour averaged annihilation cross section $\sigma\left(q_{i} \bar{q}_{i} \rightarrow\right.$ $B \bar{B})$ is calculated to lowest order in the gauge couplings, and QCD corrections are neglected. We hope this approximation shows the order of magnitude of the cross section. For $B^{0} \bar{B}^{0}$ final state the $\mathrm{Z}$ exchange is working, and $B^{+} B^{-}$pairs appear via $\gamma+Z$ exchange. This is because in the model the following Lagrangians relevant to the annihilation process emerge [1]:

$$
\begin{aligned}
L\left(B^{0}\right) & =\frac{i g}{2 \cos \theta_{W}} \partial^{\mu} B^{(0) \nu+}\left(Z_{\mu} B_{\nu}^{(0)}-Z_{\nu} B_{\mu}^{(0)}\right)+h . c . \\
L\left(B^{+} B^{-} Z\right) & =-\cos 2 \theta_{W} \cdot L\left(B^{(0)} \rightarrow B^{(+)}\right)
\end{aligned}
$$


where $B_{\mu}^{(0)}\left(B_{\mu}^{(+)}\right)$denotes the field of neutral (charged) B-particles. At $q_{j} \bar{q}_{j} Z$-vertex the usual coupling $i g \gamma_{\mu}\left(g_{V j}+g_{A j} \gamma_{5}\right)$ acts, here

$$
\left.\begin{array}{r}
g_{V j}=\frac{1}{2} \quad-\frac{4}{3} \sin ^{2} \theta_{W}, \\
g_{A j}=\frac{1}{2} \\
g_{V j}=-\frac{1}{2}+\frac{2}{3} \sin ^{2} \theta_{W}, \\
g_{A j}=-\frac{1}{2}
\end{array}\right\} j=d, c .
$$

From (2) we get for $B^{0} \bar{B}^{0}$ final states

$\sigma\left(q_{i} \bar{q}_{i} \rightarrow B^{0} \bar{B}^{0}\right)=\frac{1}{3} \frac{1}{256 \pi}\left(\frac{g}{\cos \theta_{W}}\right)^{4}\left(g_{V i}^{2}+g_{A i}^{2}\right)\left(1-\frac{4 m_{0}^{2}}{\hat{s}}\right)^{3 / 2} \frac{\hat{s}+8 m_{0}^{2}}{4 m_{0}^{4}}$.

This is decreasing at high, increasing $m_{0}$ and for $\hat{s} \gg 4 m_{0}^{2}$ it is proportional to $\hat{s} / m_{0}^{4}$ reflecting that the Lagrangian (2) is coming form a nonrenormalisable, effective model. The cross section of $B^{+} B^{-}$pairs can be expressed by (4) in the following way

$$
\begin{gathered}
\sigma\left(q_{i} \bar{q}_{i} \rightarrow B^{+} B^{-}\right)=\sigma\left(q_{i} \bar{q}_{i} \rightarrow B^{0} \bar{B}^{0} ; m_{0} \rightarrow m_{+}\right) \cdot \frac{1}{g_{V i}^{2}+g_{A i}^{2}} . \\
{\left[\left(g_{V i}^{2}+g_{A i}^{2}\right) \cos ^{2} 2 \theta_{W}+2 Q_{q_{i}} g_{V i} \sin ^{2} 2 \theta_{W} \cos 2 \theta_{W}+4 Q_{q_{i}}^{2} \sin ^{2} 2 \theta_{W}\right] .}
\end{gathered}
$$

The individual terms are due to $Z$ exchange, $\gamma-Z$ interference and $\gamma$ exchange.

We have calculated various distributions of $B \bar{B}$ pairs for $p \bar{p}$ collisions at $\sqrt{s}=1.8 \mathrm{TeV}$ and for $p p$ collisions at $\sqrt{s}=14 \mathrm{TeV}$, assuming $m_{B}=$ 400, 500, $600 \mathrm{GeV}$. Typically, at the Tevatron no notable result can be presented, however, increasing the energy to LHC, we get sizable cross sections. Also the yield of $B^{+} B^{-}$is larger than that of $B^{0} \bar{B}^{0}$. As an example we show in Fig. 1 the $\tau$-distribution of $B^{+} B^{-}$pairs at LHC, $m_{+}=400 \mathrm{GeV}$. $\frac{d \sigma}{d \tau}$ is sharply peaked after threshold $\left(4 m_{+}^{2} / s\right)$ and insignificant from about $\tau=0.1$, that is for higher invariant masses of $B^{+} B^{-}$. Fig.2 shows $\left.\frac{\partial^{2} \sigma}{\partial p_{T} \partial y}\right|_{y=0}$ as the function of the transverse momentum $p_{T}$ of $B^{+}$at vanishing rapidity $\mathrm{y}$ of $B^{+} B^{-}$for LHC, $m_{+}=400 \mathrm{GeV}$. 
For the total cross section (1) we obtain

$$
\begin{aligned}
\sigma_{\mathrm{Tev}} & =0.020(0.0145) f b \text { for } B^{+} B^{-}\left(B^{0} \bar{B}^{0}\right), m_{B}=400 \mathrm{GeV} \\
\sigma_{L H C} & =51.46 ; 15.30 ; 5.65 f b(44.35 ; 13.54 ; 4.90 f b) \\
& \text { for } B^{+} B^{-}\left(B^{0} \bar{B}^{0}\right), m_{B}=0.4 ; 0.5 ; 0.6 \mathrm{TeV}
\end{aligned}
$$

For instance, at an expected integrated luminosity of $10^{5} \mathrm{pb}^{-1}$ one gets about $5100 B^{+} B^{-}$pairs of $m_{+}=0.4 \mathrm{TeV}$ at LHC per annum.

In conclusion, we have shown that heavy $\mathrm{B}$-particle pairs have a large inclusive cross section due to $q \bar{q}$ annihilation at LHC in a suitable mass range making the detection of $B^{+, 0}$ at LHC possible.

This work is supported in part by OTKA I/7, No. 16248. 


\section{References}

[1] G. Pócsik, E. Lendvai and G. Cynolter, Acta Phys. Pol. B24, 1495 (1993); G.Cynolter, E. Lendvai and G. Pócsik, in Electroweak Symmetry Breaking, World Scientific, 1995 (Ed. F. Csikor and G. Pócsik) p. 85.

[2] G. Cynolter, E. Lendvai and G. Pócsik, Mod. Phys. Lett. A10, 2193 (1995).

[3] G. Cynolter, E. Lendvai and G. Pócsik, Mod. Phys. Lett. A9 , 1701 (1994)

[4] G. Cynolter, E. Lendvai and G. Pócsik, Acta Phys. Pol. B26, 921 (1995).

[5] S.D. Drell and T.M. Yan, Phys. Rev. Lett. 25, 316 (1970); Ann. Phys. (N.Y) 66, 578 (1971).

[6] E. Eichten, I. Hinchliffe, K. Lane and C. Quigg, Rev. Mod. Phys. 56, 579 (1984).

[7] A.D. Martin, W.J. Stirling and R.G. Roberts, Phys. Lett B 354, 155 (1995). 


\section{Figure Captions}

Fig. 1: The differential $\tau$-distribution of $B^{+} B^{-}$pairs at LHC, $m_{+}=400$ $\mathrm{GeV}$.

Fig. 2: $\left.\frac{\partial^{2} \sigma}{\partial p_{T} \partial y}\right|_{y=0}$ at LHC, $m_{+}=400 \mathrm{GeV} \cdot p_{T}$ denotes the transverse momentum of $B^{+}$and $y$ is the rapidity of $B^{+} B^{-}$. 
Fig.1.

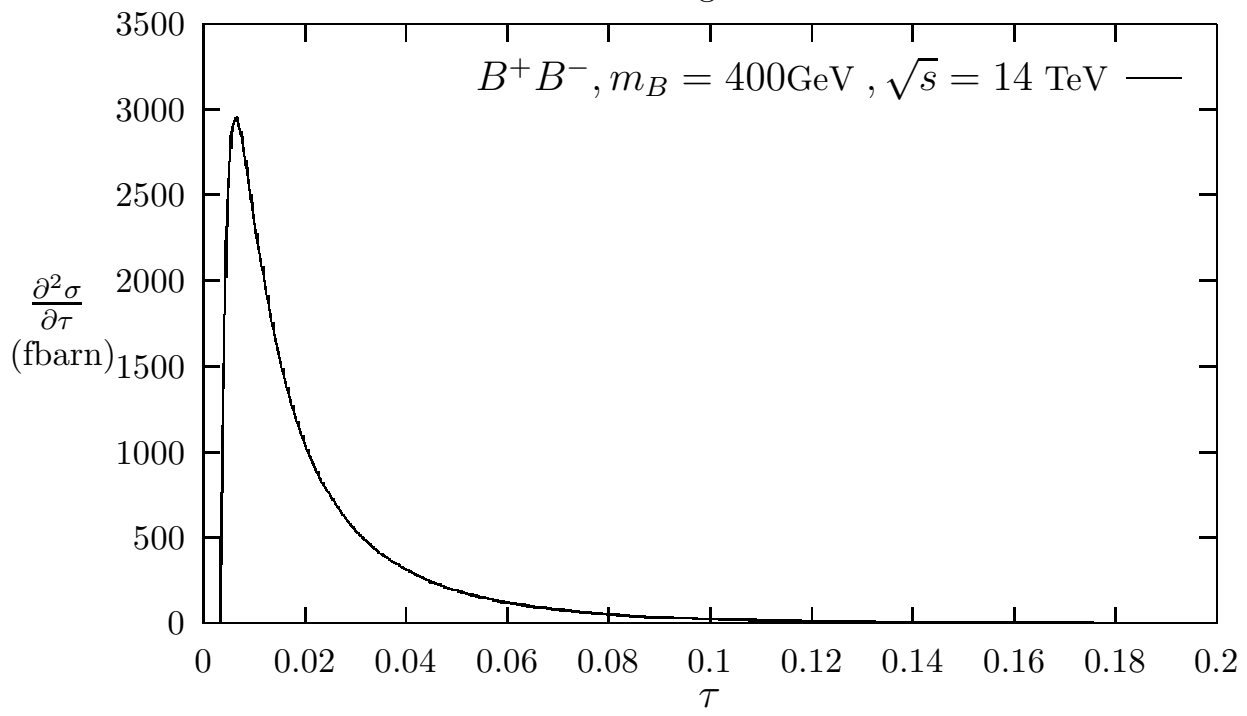

Fig.2.

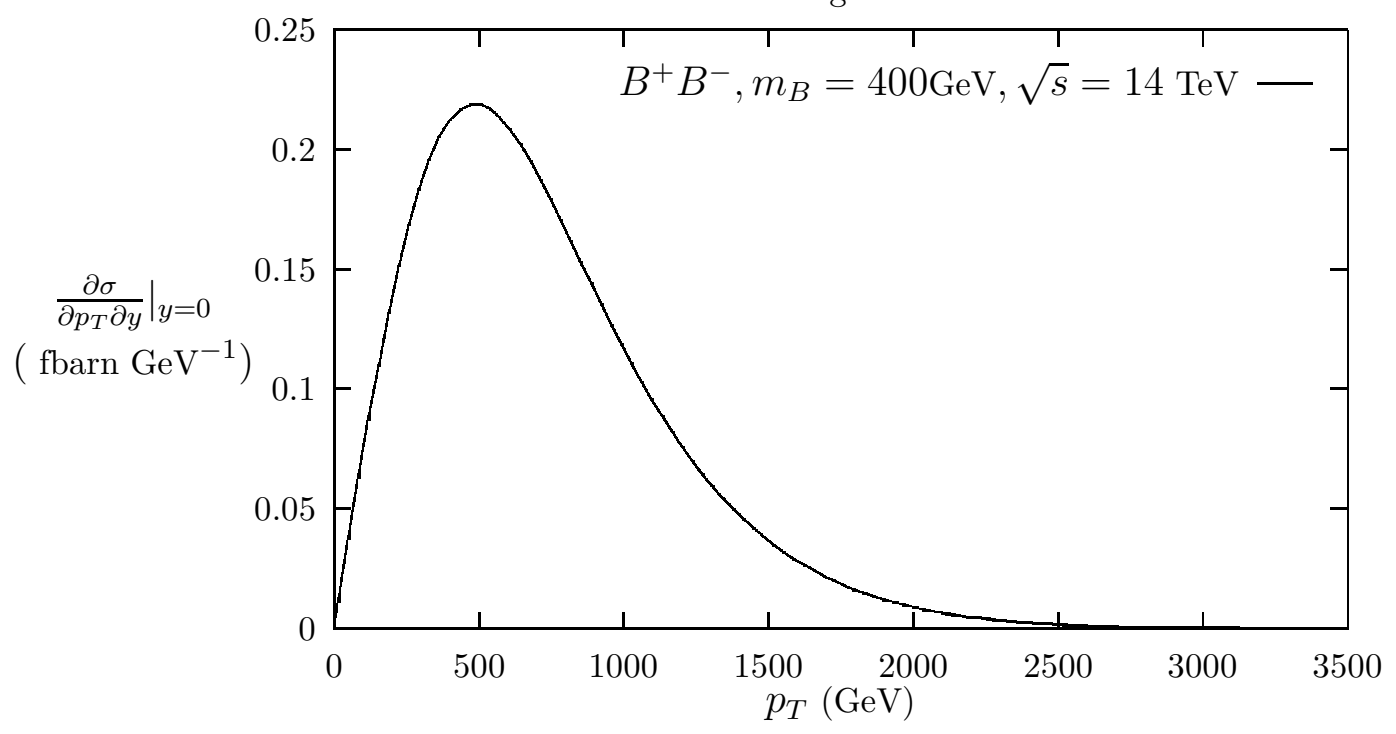

